\title{
In situ S/TEM Observation of Hydrogen Bubbles Formation and Evolution in Aluminium Nanoparticles
}

Yang $\mathrm{Liu}^{1,2}$ and Ting $\mathrm{Zhu}^{3}$

${ }^{1 .}$ Department of Materials Science and Engineering, North Carolina State University, Raleigh, North Carolina 27695, USA

2. Analytical Instrumentation Facility, North Carolina State University, Raleigh, North Carolina 27695, USA

3. Woodruff School of Mechanical Engineering, Georgia Institute of Technology, Atlanta, Georgia 30332, USA

The formation of voids and successive growth of hydrogen bubbles in solid metals have attracted extensive attention, mainly because the existence of such bubbles and/or blisters can significantly deteriorate the mechanical properties of the host metals $[1,2]$. Various kinds of treatments have been found to be able to introduce cavity, bubbles or blisters in different metals, such as high energy/high dose proton implantation, quenching in $\mathrm{H}_{2} / \mathrm{H}_{2} \mathrm{O}$ gas, and cathodic reaction [1, 3, 4]. However, the initiation of cavity, dynamic evolution of bubbles/blisters, and how the mechanical properties of metals decay are still not well understood. In this work, we employ in situ environmental scanning/transmission electron microscopy (S/TEM) experiments to investigate the formation and evolution of hydrogen bubbles in Aluminium (Al) nanoparticles (NPs) dynamically.

The in situ experiments were carried out in Protochips Atmosphere ${ }^{\mathrm{TM}}$ TEM environmental gas cell in a probe aberration corrected FEI Titan G2 60-300 kV S/TEM operated under $200 \mathrm{keV}$. High purity 5\% $\mathrm{H}_{2}$ (with balancing gas Ar) was introduced into the cell as hydrogen source. The gas pressure in the environmental cell could be controlled from 1 Torr to 700 Torr by using Atmosphere ${ }^{\mathrm{TM}}$ manifold. Under the irradiation from high energy electron beam, hydrogen molecules were ionized to generate atomic $\mathrm{H}$ to facilitate its entrance through the surface native $\mathrm{Al}_{2} \mathrm{O}_{3}$ layer on $\mathrm{Al}$ NPs. Once the cavity at the interface of $\mathrm{Al}_{2} \mathrm{O}_{3}$ shell and $\mathrm{Al}$ core has nucleated, hydrogen gas bubbles grow by absorbing more hydrogen atoms, which is very similar with the hydrogen bubbles formation in Al pillar as reported in ref [5].

Figure 1 shows the STEM high-angle annular dark-field (HAADF) images from a cluster of Al NPs before and after exposure to hydrogen environment, indicating the formation of hydrogen bubbles at the $\mathrm{Al}_{2} \mathrm{O}_{3} / \mathrm{Al}$ interface. This phenomenon is also confirmed in the bright field TEM imaging, as shown in Figure 2a. After continuing beam irradiation on Al NPs in hydrogen environment, the size of hydrogen bubbles keep increasing and the neighbouring bubbles merge to bigger bubbles. Moreover, the Al core starts to become porosity, as shown in Figure $2 \mathrm{~b}$. The generation of porosity inside solid Al core can be attributed to the formation of $\mathrm{H}$-vacancies clusters, and the aggregation of these clusters to form pores inside the solid Al. The effects of beam intensity, hydrogen pressure, and size of NPs on the evolution of hydrogen bubbles and porosity will also be discussed. 


\section{References:}

[1] J.B. Condon and T. Schober, Journal of Nuclear Materials 207 (1993), 1.

[2] D.E.J. Talbot, International Metallurgical Reviews, 20 (1975) 166.

[3] S.K. Das and M. Kaminsky, Radiation Effects on Solid Surfaces, Chapter 5, pp 112-170.

[4] P. Rozenak, International Journal of Hydrogen Energy 32 (2007) 2816.

[5] D.-G. Xie et al, Nature Materials 14 (2015), 899.

[6] This work was performed in part at the Analytical Instrumentation Facility (AIF) at North Carolina State University, which is supported by the State of North Carolina and the National Science Foundation (award number ECCS-1542015). The AIF is a member of the North Carolina Research Triangle Nanotechnology Network (RTNN), a site in the National Nanotechnology Coordinated Infrastructure (NNCI).
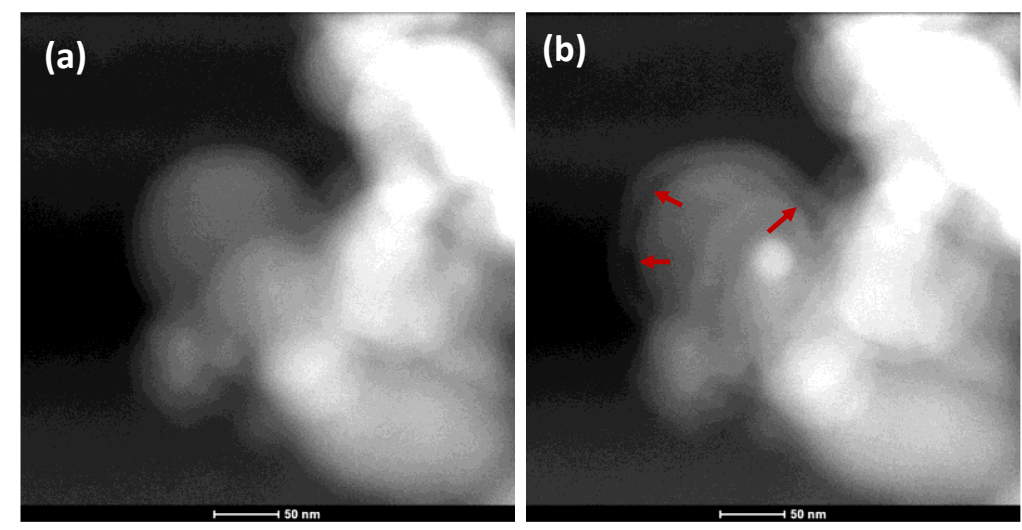

Figure 1. HAADF images of a cluster of Al NPs before (a) and after (b) the formation of hydrogen bubbles.
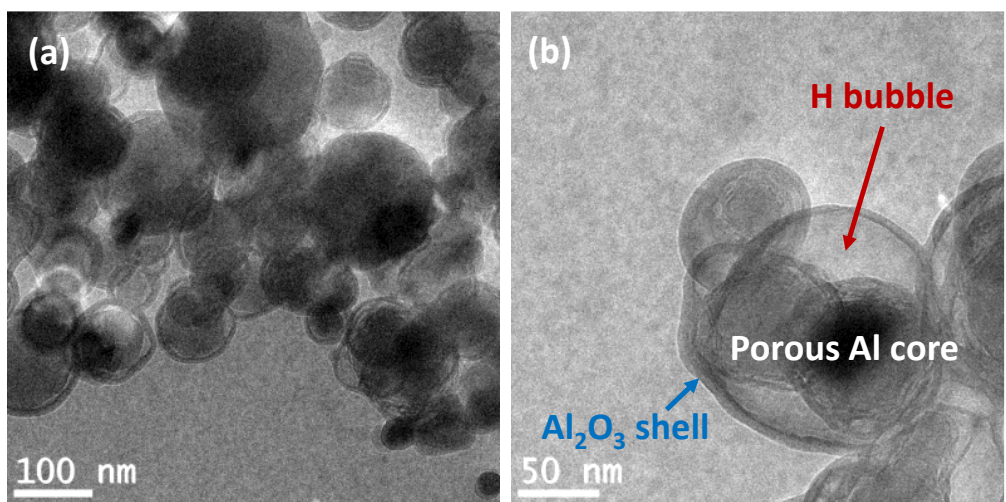

Figure 2. (a) TEM image showing the formation of hydrogen bubbles at the interface of surface $\mathrm{Al}_{2} \mathrm{O}_{3}$ shell and inner Al core in each individual NPs. (b) the morphology of Al NPs after intensive beam irradiation in hydrogen environment, showing the hydrogen bubbles and the porosity of inner $\mathrm{Al}$ core. 